\title{
Experimental and Numerical Studies of Natural Convection on Elliptical Tube in Thermal Asymmetric Cavity
}

\author{
Angga Tegar Setiawan, Budi Utomo Kukuh Widodo* Nila Rahmawati \\ Department of Mechanical Engineering, ITS, Sukolilo, Surabaya 60111, Indonesia \\ Received: 2 July 2018, Revised: 3 August 2018, Accepted: 15 August 2018
}

\begin{abstract}
The characteristic of natural convection heat transfer in a thermally asymmetric cavity is influenced by temperature differences and space between the heat source and cavity wall. The heat source is any bluff body placed inside the cavity. This research used a heated elliptic tube as the heat source inside the cavity. The three tubes are of 2.5 aspect ratio (AR) in a vertically aligned arrangement. The cavity was thermally asymmetric because three sides of the cavity were insulated while the other side was exposed to convection with ambient air. The clearance ratio (CR) as the ratio between the gap from the insulated wall to the major axis of the tubes to the space of the cavity is varied 0.4 and 0.6 . while the pitch ratio is constant at 3.5a. this study analyzes the characteristics of heat transfer from the surface of the elliptical tube to the surrounding air experimentally and numerically. The result reveals that the highest heat transfer coefficient 0 f $0.4 \mathrm{CR}$ is higher than that of $0.6 \mathrm{CR}$. The numerical solution shows the contour and streamlines of the fluid flow in all conditions as the characteristics of natural transfer thermally asymmetric cavity.
\end{abstract}

Keyword : Free convection, Elliptical tube, Clearance ratio, Asymmetric termal

\section{Introduction}

Natural convection is an important heat transfer phenomenon in designing heat exchanger. One application of natural convection heat transfer widely used in daily life is a refrigerator condenser. Refrigerator condenser serves as a heat exchanger that can convert the form of refrigerant from high pressure and temperature into a liquid form.

One type of condenser used in a refrigerator is a hot wall condenser. It consists of a circular tube which is located on the inside of the right and left the wall of the refrigerator. It is in direct contact with the refrigerator wall plate. A lot of effort has been made to film condensation mechanism on the horizontal tube. Most of the studies conducted to focus on techniques for increasing extended surfaces that can increase heat transfer, not only from a larger ratio of surface area and tube volume but also because of the use of surface tension from thinner layers of condensate. Another alternative is to use noncircular tube shape which serves to thin condensate film. Not only through surface tension effects but also through an increased effect of gravity as a result of placing a larger proportion of the condensing surface in line with vertical [1].

Natural convection heat transfer performance on smaller axis ratios results in higher heat transfer rates. This shows that natural convection heat transfer on the elliptical tube is better than the circular tube [2]. Another study conducted by Ashjaee, et al [3] also concluded

\footnotetext{
*Email:email: buditem@me.its.ac.id phone: +62 81520955877
}

that a decrease in the tube axis ratio can increase Nusselt number. T. Yousefi and M. Ashjaee [4] conducted an experimental study of natural convection in the elliptical tube isotherm arrangement with the position of the vertical major axis. The experiment was carried out using a Mach-Zehnder interferometer with two to five cylinders and Rayleigh numbers ranging between 103 and $2.5 \mathrm{x}$ 103 analyzing the effect of cylinder distance and Rayleigh number. Form the results of the study it can be concluded that heat transfer from cylinder downstream increasing by increasing cylinder distance.

T. Yousefi, et al. [5] conducted an experimental study of two -dimensional natural convection with an arrangement of five elliptical tubes arranged arrays on the main vertical axis and bounded by two adiabatic walls. Indicates that the Nusselt number is maximum for tube 1-3 at a certain wall space. However, for tubes 4-5 the maximum heat transfer is at infinite wall distance. In addition to Rayleigh numbers, there is an optimal distance where the heat transfer in the array arrangement is maximum. At the optimal wall distances there is about a $10 \%$ increase in heat transfer compared to a tube that does not have a dividing wall.

Nada, et al. [6] conducted an experimental and numerical study to analyze heat transfer and flow characteristics that crossed the tube surface at different angles. The result showed that the orientation of the tube at angles of attack zero showed the maximum Nusselt number. Hyun Woo Cho, et al. [7] conducted a numerical study 
of two-dimensional natural convection with one elliptical tube and one circular tube placed in the enclosure. It shows that in the case of the elliptical tube position above the circular tube there are two primary vortices produced in the left and right enclosures with two secondary eddies on the upper wall except for the case of AR 7.5 and 1.00. Massimo C. and Emanuele H. [8] conducted a numerical study of natural convection on the elliptical tube by developing SIMPLE-C algorithm computer-code with uniform tube surface temperature. Shows that the increase in the Nusselt numbers averages along with the increase in the Prandtl number and the decrease in the gradient. In addition, the decrease in the axis ratio causes an increase in Rayleigh numbers and an increase in the orientation angle of the tube causes a decrease in the Nusselt number.

Wael M. E. [9] conducted a numerical study and natural convection experiments on an elliptical tube with the axis ratio 0.496. Shows that For flow, the maximum local Nusselt number occurs at the point of lower stagnation in the range of Reynolds numbers. For opposite flow, the maximum local Nusselt number occurs at the highest stagnation point for high Reynolds numbers ( $\mathrm{Re}=1100$ and 1306) then transferred to the lower stagnation point with a low Reynolds number $\mathrm{Re}=390$ because natural convection is a heat transfer mechanism at low Reynolds numbers

Therefore, the elliptical tube can be an alternative to increasing heat transfer that occurs in the refrigerator condenser. By conducting further research to analyze the characteristics of natural convection heat transfer in the elliptical tube in the thermal asymmetry cavity is compatible with the hot wall condenser refrigerator model with a variation of the clearance ratio (CR).

\section{Experimental Method}

The object studied consisted of three elliptical tubes arranged vertically with the major axis tube with one another in a line and parallel to the vertical axis. The tool installation scheme is shown in Figure 1 . The tube arrangement is placed in a closed cavity to support natural convection conditions. Closed cavities are made of acrylic material which has a high melting point, which is $160^{\circ} \mathrm{C}$ and is colorless (transparent) so that observation and installation of test equipment will be easier to do.

The cavity has $225 \mathrm{~mm}$ in height. The width (y) of the cavity is $100 \mathrm{~mm}$ with the distance between tube arrangement to the insulated wall $(\mathrm{x})$ equals $0.4 \mathrm{y}$ and $0.6 y$. Pitch ratio elliptical tube $3.5 \mathrm{a}$ with $\mathrm{a}=21.5 \mathrm{~mm}$ (a half of major diameter). The study uses an electric heater installed inside each tube so that the tube can function as a heat source. To control the temperature of the heater, a thermocontrol is used. The surface of each tube will be fitted with 6 thermocouple cables, 10 thermocouple cables within the cavity, and 3 thermocouple cables on the outer wall. Overall, there are 31 temperature data retrieval po-

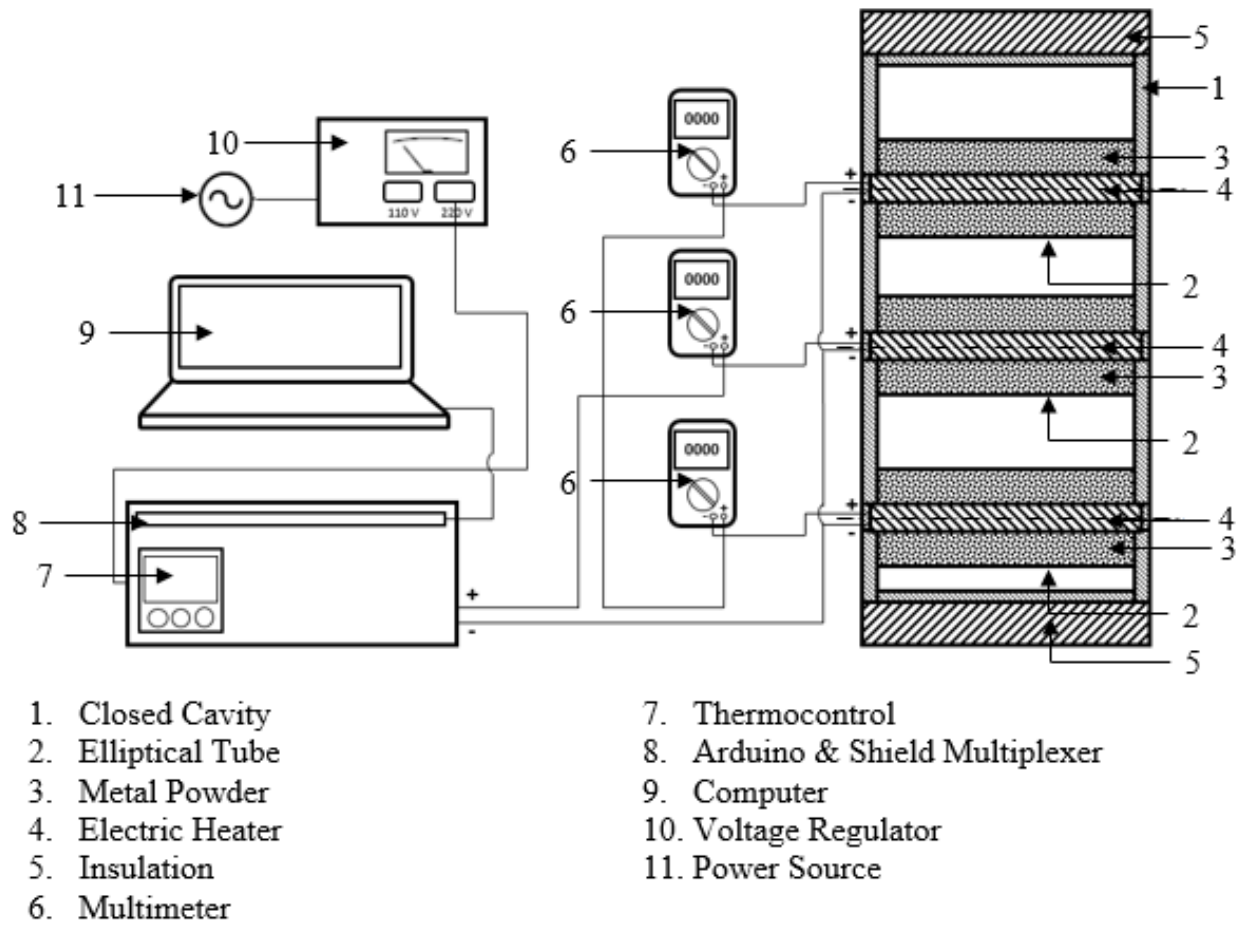

Figure 1. Installation Scheme 


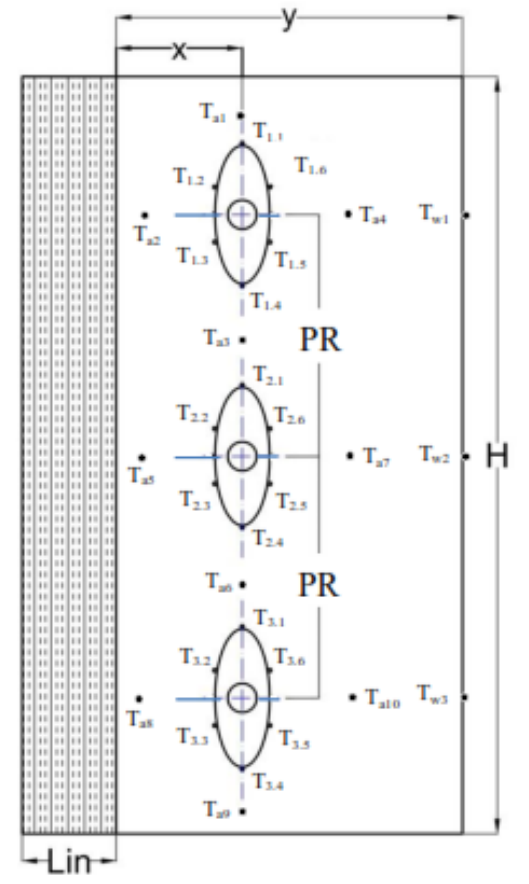

Figure 2. Scheme for placing data collection points

ints. The scheme for placing data collection points can be seen in Figure 2, One thermocouple cable of the same type is also used as a reference thermocouple. it is affixed to the surface of one tube and connected to thermocontrol. Data acquisition is done using Arduino microcontroller and data is displayed on Microsoft Excel worksheets helped by PLX-DAQ software.

Natural convection occurs when the fluid moves due to the buoyancy force, which is the fluid motion that occurs due to differences in density. Changes in fluid density are influenced by temperature differences. Increasing temperature causes a decrease in fluid density due to fluid expansion $(\partial \rho / \partial \mathrm{T}<0)$. Differences in density in terms of temperature differences require properties that represent variations in the density of liquids with temperatures at constant pressure, namely $\beta$, which is defined as the volume expansion coefficient. In an ideal gas, the volume expansion coefficient $(\beta)$ at temperature $\mathrm{T}$ is equivalent to the opposite of absolute temperature. The equation volume expansion coefficient $(\beta)$ can be shown as follows.

$$
\beta=\frac{1}{T}
$$

The Nusselt number $(\mathrm{Nu})$ correlation to determine the natural convection heat transfer coefficient is as follows:

$$
N u=\frac{h L}{k}=C\left(G r_{L} P r\right)^{n}=C R a_{L}^{n}
$$

With $\mathrm{Ra}_{L}^{n}$ is Rayleigh number which is the multiplication between Grashof (Gr) and Prandtl (Pr) number. The Grashof number describes the relationship between Buoy- ancy and fluid viscosity while the Prandtl number states the ratio of diffusivity momentum (kinematic viscosity) and thermal diffusivity. Rayleigh number is expressed in the following equation:

$$
R a_{L}=G r_{L} \operatorname{Pr}=\frac{g \beta\left(T_{s}-T_{\infty}\right) L_{c}^{3}}{\nu^{2}} \operatorname{Pr}=\frac{g \beta\left(T_{s}-T_{\infty}\right) L_{c}^{3}}{\nu \alpha}
$$

At the length of the characteristic L of geometry. For laminar and turbulent flows all properties were evaluated at film temperature.

$$
T_{f} \equiv \frac{T_{s}+T_{\infty}}{2}
$$

\section{Numerical Method}

By definition, computational fluid dynamics (CFD) is the study of how to predict fluid flow, heat transfer, chemical reactions, and other phenomena by solving mathematical equations. CFD actually replaces partial differential equations of continuity, momentum, and energy with algebraic equations. In general, there are three stages that must be done in CFD simulations [10], namely:

\subsection{Pre-processing}

Pre-processing is the first step in building and analyzing a CFD model. The technique is to create a model in a CAD (Computer Aided Design) package, create a mesh that is suitable, then apply boundary conditions and other properties. Meshing is the process of breaking domains into smaller volumes. This is to facilitate stream domain discretion and apply equations that govern the flow domain. Simulations are obtained accurately if selection of meshing on geometry is made following the predicted changes in flow patterns that occur.

Preprocessing consists of making geometry, meshing, modeling and determining boundary conditions. Before performing calculations, geometry and mesh (computational domain) are created using GAMBIT 2.4.6 software. After computational geometry is created using GAMBIT. Then the appropriate boundary condition value is entered. The boundary condition scheme and mesh structure for the enclosure and elliptical tube are shown in Figure 3, as follows

Grid independence test is done to determine the number of mesh that is good/optimal to use in the simulation process. The independence grid test can be done by determining the number of cells that you want to experiment with to get a good number of cells. from the results of the grid independent test, there is an adequate number of nodes of 22500 nodes.

\subsection{Processing}

Processing (core solution finder program) CFD calculates the conditions applied during pre-processing. Some of the arrangements to be made include the models, ma- 

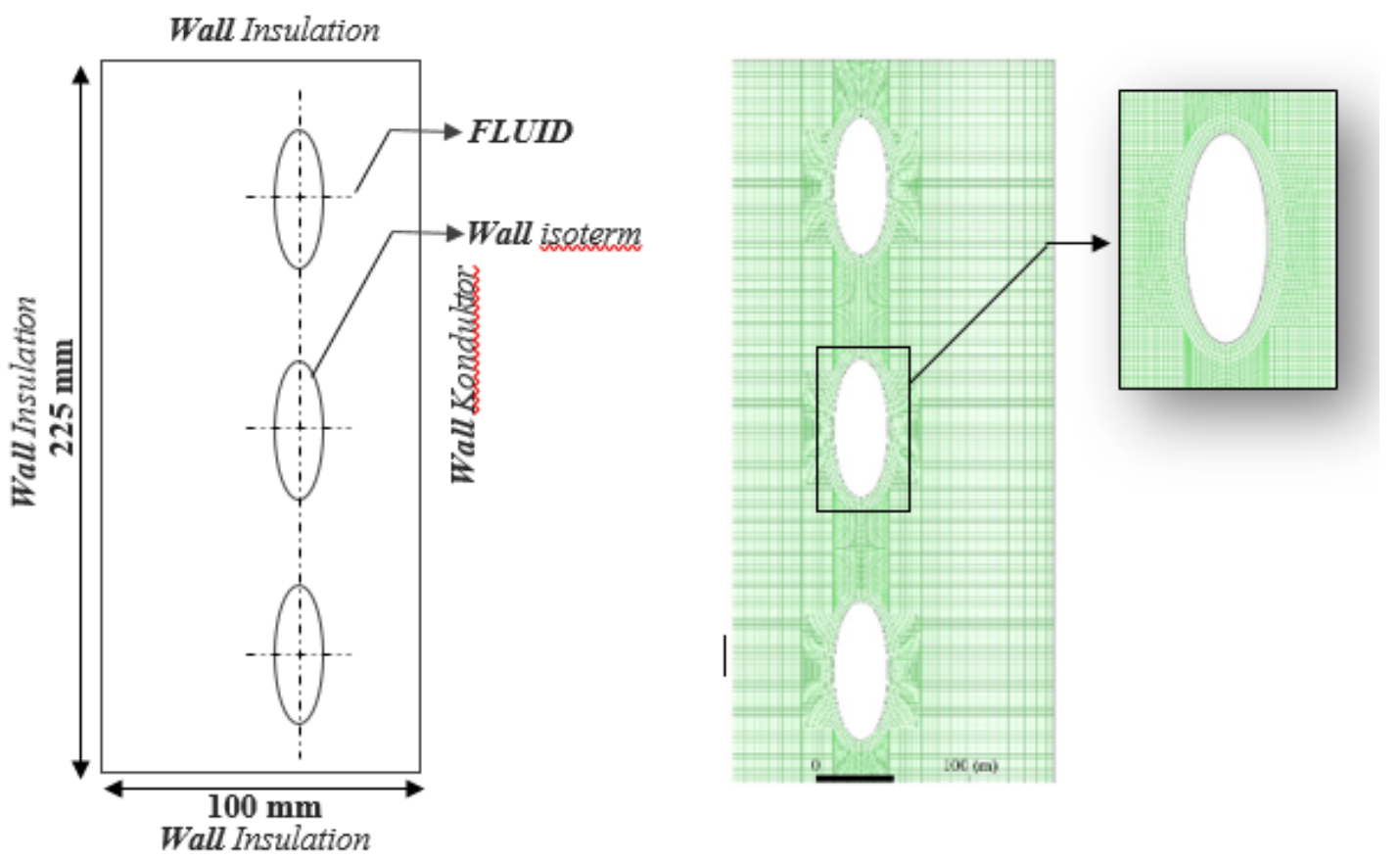

Figure 3. Boundary Condition and Meshing of Geometri

terials, boundary conditions, operating conditions, and initialize conditions. In the processing stage complete the governing equations. Such as the completion of partial differential equations carried out by Wael M. E. [9] using mass, momentum, and energy conservation equations. For incompressible flow, continuity and momentum are resolved for the velocity and pressure distribution in the computational process. The $2 \mathrm{D}$ continuity equation used is.

$$
\frac{\partial u}{\partial x}+\frac{\partial v}{\partial y}
$$

The momentum equation in the $\mathrm{x}$-direction can be written as follows:

$\rho\left[u \frac{\partial u}{\partial x}+v \frac{\partial u}{\partial y}\right]=-\frac{\partial p}{\partial x}-\beta \rho g\left(T-T_{\infty}\right)+\pi\left[\frac{\partial^{2} u}{\partial x^{2}}+\frac{\partial^{2} u}{\partial y^{2}}\right]$

The momentum equation in the y-direction can be written as follows:

$\rho\left[u \frac{\partial v}{\partial x}+v \frac{\partial v}{\partial y}\right]=-\frac{\partial p}{\partial x}-\beta \rho g\left(T-T_{\infty}\right)+\pi\left[\frac{\partial^{2} v}{\partial x^{2}}+\frac{\partial^{2} v}{\partial y^{2}}\right]$

Energy equation:

$$
\rho C_{p}\left[u \frac{\partial T}{\partial x}+v \frac{\partial T}{\partial y}\right]=k\left[\frac{\partial^{2} T}{\partial x^{2}}+\frac{\partial^{2} T}{\partial y^{2}}\right]
$$

\subsection{Post Processing}

Post processing is the last step in analyzing CFD. This step is to organize and interpret the CFD simulation data that can be presented in the form of images, curves, and animations. Post processing is increasingly advanced and developing so that it has considerable graphics and visualization capabilities. The tools provided by Fluent for visualizing are Domain geometry, display, Vector plot, Contour plot, and 2D surfaces plot.

\section{Results and Discussion}

\subsection{Temperature Distribution}

From the research that has been done, it is obtained temperature data at 31 points including 18 points on 3 elliptical tubes, 10 points on cavity and 3 points on uninsulated walls. The research data was obtained from each variation of $\mathrm{CR}$ at $\mathrm{PR} 3.5 \mathrm{a}$ with reference temperature $55^{\circ} \mathrm{C}$. as shown in Figure 3. From Figure 4 it can be seen that the temperature distribution occurs in each tube in the thermal asymmetry cavity. In each tube shows the same trend at the highest temperature always occurs at $\theta$ $=0^{\circ}$ or $\theta=360^{\circ}$ then decreases at the lowest temperature at $\theta=180^{\circ} \mathrm{As}$ in tube 1 the surface temperature increases higher when compared to tube 2 and 3 . Likewise in tube 2 , it tends to be higher in surface temperature when compared to tube 1 . This also occurs in variations in CR 0.4 and 0.6.

This pattern can occur in natural convection heat transfer for tube arrangement because in this arrangement there will be interaction of heat transfer between tubes. The lower tube (tube 3) does not have the effect of heat transfer from the previous tube (upstream tube) so that it has the characteristics of a single tube. Whereas for tubes 2 and 1 get the influence of the previous tube heat transfer (upstream tube). The heat from the upstream tube will move into the air which will then come into contact with 

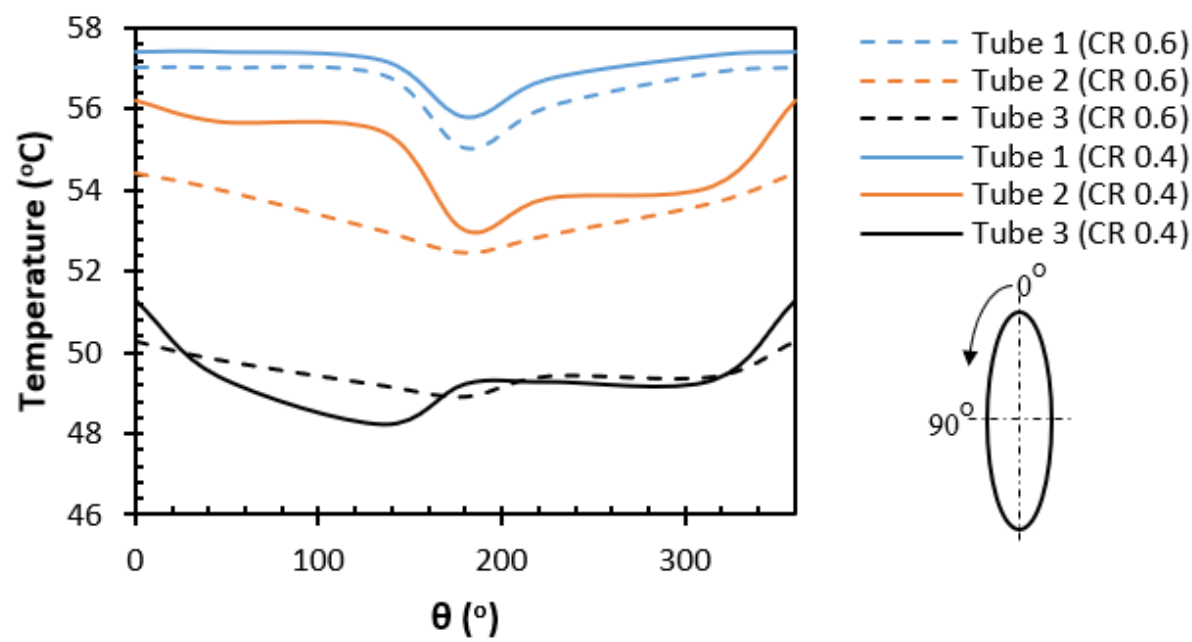

Figure 4. Graph of temperature distribution

the downstream tube. Heat transfer will also occur in the downstream tube but not as big as what happens in the lower tube. This interaction between the tubes causes a difference in the convection heat transfer of each tube in the arrangement.

In addition, the lowest temperature that occurs at $\theta$ $=180^{\circ}$ and the highest temperature occurs at $\theta=0^{\circ}$ this can happen because at $\theta=180^{\circ}$ is the point of buoyant flow stagnation so that at that point the greatest heat transfer occurs. Conversely, at $\theta=0^{\circ}$ buoyant flow leaves the tube so that the heat transfer that occurs is smaller. This also occurs in research conducted by T. Yousefi \& M. Ashjaee [4] which is caused by the plume of the lower tube affecting the heat transfer characteristics in two different ways. The first, behaves as a forced convection field for the upper cylinder and, second, it causes a lower temperature difference between the cylinder surface and the incoming air. From the graph above, it can be seen that the effect of $\mathrm{CR}$ variation on the temperature on the surface of the elliptical tube has a difference in temperature difference. In CR 0.4 the difference in temperature between tube 1 and the others tends to be greater when compared to CR 0.6. Tube 1 and 2 surface temperatures in CR 0.4 tend to be higher than CR 0.6. In addition, the surface temperature of tube 3 in CR 0.4 and 0.6 tends to be the same.

\subsection{Coefficient of Convection on Elliptical Tube}

From the result of the calculations that have been done, the local convection coefficients obtained in each segment in all variations can be shown in Table 1 as follows. From the convection coefficient data on table 1, a graph of the relationship between the tube and convection coefficient above is shown as shown in Figure 5, it is obtained the convection coefficient relationship between tube and $\mathrm{CR}$. The lowest coefficient value is in the range $\theta=180^{\circ}$. and the highest convection coefficient values tend to be in the vertical segment. The same is true of re-

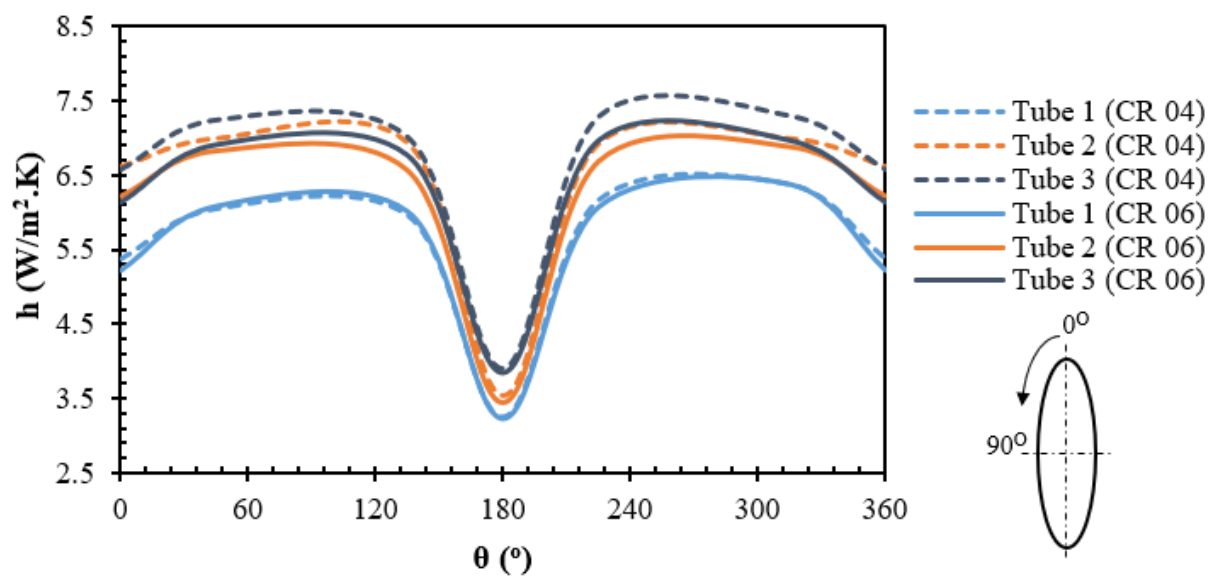

Figure 5. Graph of convection coefficient on tube surface 
Table 1. Convection Coefficient Value in Each Segment at PR 3.5a

\begin{tabular}{llll}
\hline Tube & $\left.\theta \mathbf{(}^{\circ}\right)$ & \multicolumn{2}{c}{$\mathbf{h}\left(\mathbf{W} / \mathbf{m}^{2} \cdot \mathbf{K}\right)$} \\
\cline { 3 - 4 } & & $\mathbf{C R ~} \mathbf{0 . 4}$ & $\mathbf{C R ~ \mathbf { 6 . 6 }}$ \\
\hline Tube 1 & 0 & 5.38 & 5.21 \\
& 45 & 6.05 & 6.08 \\
& 135 & 5.96 & 6.00 \\
& 180 & 3.24 & 3.23 \\
& 225 & 6.17 & 6.07 \\
Tube 2 & 315 & 6.38 & 6.37 \\
& 0 & 6.63 & 6.22 \\
& 45 & 7.01 & 6.83 \\
& 135 & 6.95 & 6.57 \\
& 180 & 3.54 & 3.44 \\
Tube 3 & 225 & 6.94 & 6.69 \\
& 315 & 7.01 & 6.89 \\
& 0 & 6.58 & 6.14 \\
& 45 & 7.25 & 6.92 \\
& 135 & 7.02 & 6.77 \\
& 180 & 3.90 & 3.85 \\
& 225 & 7.30 & 6.98 \\
& 315 & 7.30 & 6.97 \\
\hline
\end{tabular}

search conducted by H. M. Badr [2] where $\mathrm{Nu}$ is the maximum average on the vertical axis. That $\mathrm{Nu}$ maximum point is always near $180^{\circ}$, while $\mathrm{Nu}$ minimum point is at a stagnation rate in all cases. Based on the graph, it is found that the convection coefficient value increases from tube 1 to tube 3. For the same set point, it.

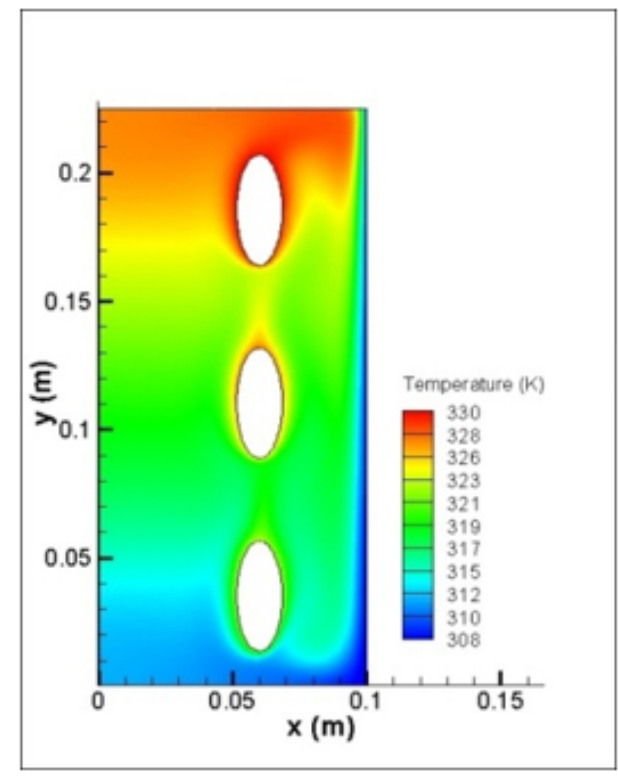

(a)

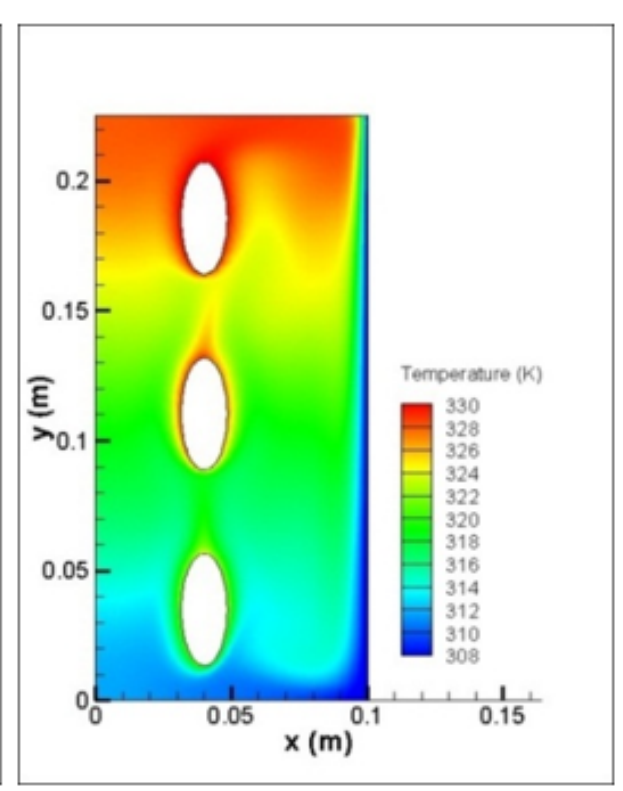

(b)

Figure 6. Temperature distribution contours (a) CR 0.6 (b) CR 0.4 


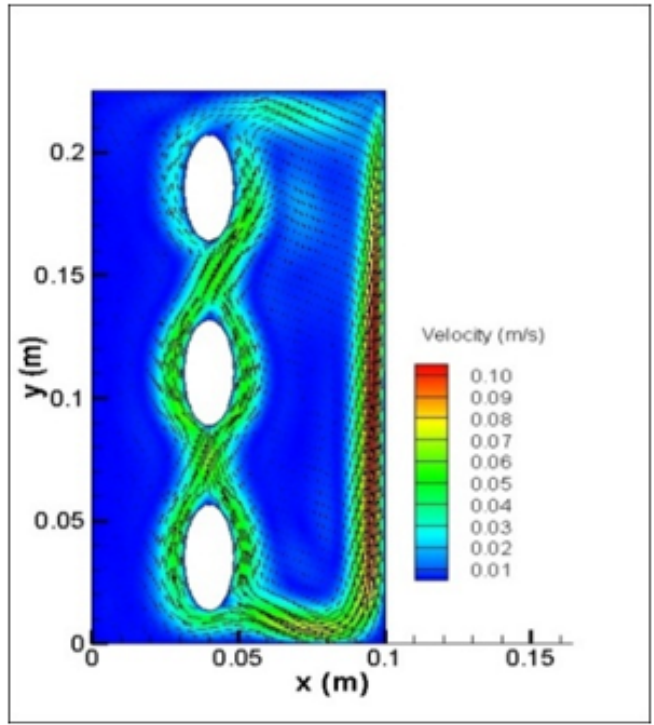

(a)

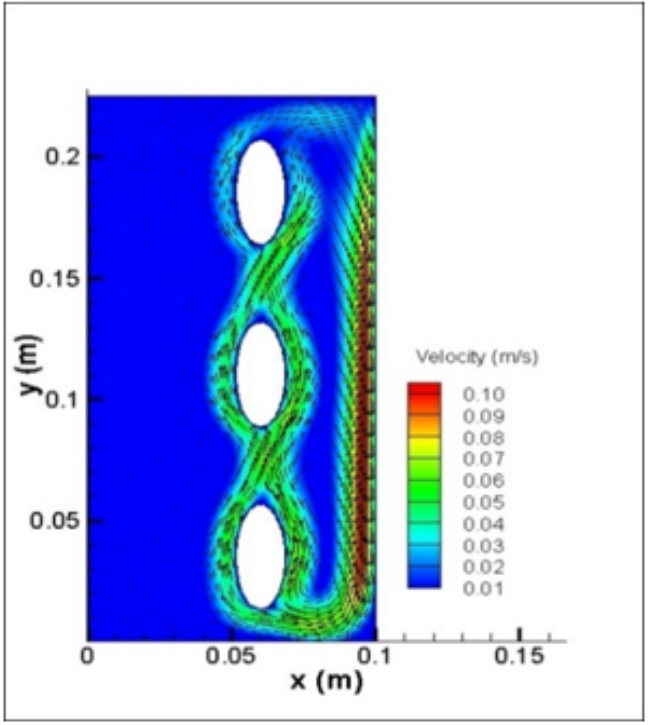

(b)

Figure 7. Velocity vector and contour (a) CR 0.4 , (b) CR 0.6

the study shows different temperature gradients. The temperature gradient in CR 0.6 and CR 0.4 shows the highest temperature of the cavity at the upper side of the cavity. This is caused by bouyancy force where the higher the fluid temperature, the lower the density. So that the area above the cavity has a higher temperature. Based on the research conducted by T. Yousefi et. al. [5] who conducted an experiment on natural convection heat transfer of the elliptical tube arrangement with variations in the width of the slit, obtained a smaller gap width, the convection coefficient value will be smaller. This happens because the narrower width of the gap causes the interaction between the wall and the bouyant force to limit the air flow formed. so this shows that CR 0.4 tends to have good natural convection displacement compared to CR 0.6. this is because the width of the gap between the tube and the conduction wall is greater.

\subsection{Velocity Vector}

From figure 7 the above contour velocity shows that the highest speed occurs around the conductor wall. This shows that the temperature difference that occurs in the area is relatively high and this also occurs in all variations. So this also indicates that the highest heat transfer occurs on the wall of the conductor. In addition, the fluid also moves around the elliptical tube in the direction of fluid velocity that occurs upward. This indicates that the Buoyance force effect is clearly seen by the velocity vector that occurs around the tube surface.

\section{Conclussions}

From the results of experimental and numerical studies that have been carried out on natural convection heat transfer elliptical tubes installed in the asymmetry cavity it can be concluded that CR 0.4 has better natural convection heat transfer compared to CR 0.6. This is also shown in the contour of the cavity temperature distribution in $\mathrm{CR}$ 0.6. In addition, based on CR speed vectors in CR 0.4 tend to be higher when compared to CR 0.6. this shows that the high-temperature difference in CR 0.4 causes the fluid to move faster so that natural convection heat transfer tends to be better.

\section{Acknowledgments}

This research was funded by LPPM (Lembaga Penelitian dan Pengabdian Masyarakat)

\section{References}

[1] S. Memory, V. Adams, and P. Marto, "Free and forced convection laminar film condensation on horizontal elliptical tubes," International journal of heat and mass transfer, vol. 40, no. 14, pp. 3395-3406, 1997.

[2] H. Badr, "Laminar natural convection from an elliptic tube with different orientations," 1997.

[3] M. Ashjaee, M. Amiri, B. Baghapour, and T. Yousefi, "An empirical correlation for natural convection from confined elliptic tube," Experimental heat transfer, vol. 20, no. 3, pp. 213-228, 2007.

[4] T. Yousefi and M. Ashjaee, "Experimental study of natural convection heat transfer from vertical array of isothermal horizontal elliptic cylinders," Experimental Thermal and Fluid Science, vol. 32, no. 1, pp. 240-248, 2007.

[5] T. Yousefi, M. Paknezhad, M. Ashjaee, and S. Yazdani, "Effects of confining walls on heat transfer from a vertical array of isothermal horizontal elliptic 
cylinders," Experimental Thermal and Fluid Science, vol. 33, no. 6, pp. 983-990, 2009.

[6] S. Nada, H. El-Batsh, and M. Moawed, "Heat transfer and fluid flow around semi-circular tube in cross flow at different orientations," Heat and mass transfer, vol. 43, no. 11, p. 1157, 2007.

[7] H. W. Cho, Y. G. Park, and M. Y. Ha, "The natural convection in a square enclosure with two hot inner cylinders, part i: The effect of one elliptical cylinder with various aspect ratios in a vertical array," International Journal of Heat and Mass Transfer, vol. 125, pp. 815-827, 2018.
[8] M. Corcione and E. Habib, "Multi-prandtl correlating equations for free convection heat transfer from a horizontal tube of elliptic cross-section," International Journal of Heat and Mass Transfer, vol. 52, no. 5-6, pp. 1353-1364, 2009.

[9] W. M. El-Maghlany, M. A. Alnakeeb, M. A. Teamah, and M. M. Sorour, "Experimental and numerical study of laminar mixed convection from a horizontal isothermal elliptic cylinder," International Journal of Thermal Sciences, vol. 130, pp. 116-127, 2018.

[10] T. Firman, "Dasar-dasar cfd menggunakan fluent," Informatika Bandung, Bandung, 2008. 